\title{
A Multi-Target Tracking and Detection Algorithm for Wireless Sensor Networks
}

\author{
Gang Wang \\ Hubei Open University, Hubei, Wuhan430074, China

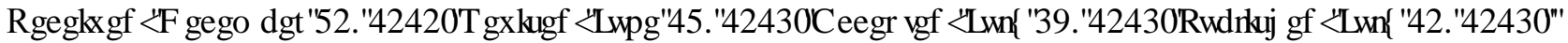

\begin{abstract}
There are a large number of sensor nodes in wireless sensor network, whose main function is to process data scientifically, so that it can better sense and cooperate. In the network coverage, it can comprehensively collect the main information of the monitoring object, and send the monitoring data through short-range wireless communication to the gateway. Although there are many applications in WSNs, a multi-Target tracking and detection algorithm and the optimization problem of the wireless sensor networks are discussed in this paper. It can be obviously seen from the simulation results that this node cooperative program using particle CBMeMBer filtering algorithm can perfectly handle multi-target tracking, even if the sensor model is seriously nonlinear. Simulation results show that the tracking - forecasting data association scheme applying GMCBMeMBer, which is proposed in this paper, runs well in identifying multiple target state, and can improve the estimation accuracy of multiple target state.
\end{abstract}

Keywords - wireless sensor networks; nodes in sleep mode; detection probability; target signal

\section{INTRODUCTION}

$\mathrm{I}_{\mathrm{i}}^{\mathrm{n}}$ $\mathrm{n}$ recent years, moving multi-target tracking (MMTT) implementing by WCSN has gained more and more attention[1-2]. The difficulty of MMTT lies in mastering the unknown and varying target numbers, and their status in clutter environment [3-5]. The probability hypothesis density (PHD) filter based on finite set statistics (FISST) [6] is obtained from the recursion of first order matrix [7] propagation. The degradation of PHD filter performance can be solved by highend target number. This CPHD filter is a solution [8], but it is inconvenient to use because of its high computational complexity. Base balanced multiple target multi-Bernoulli (CBMeMBer) filtering algorithm [9] provides a solution of easy handling, through spreading the multi-Bernoulli RFS parameters similar to multi-objective RFS, while ensuring the unbiased intensity function. Both particle filter and Gaussian compound filter are implementation schemes[10]. PCBMeMBer filtering algorithm is applied to realize the node cooperative scheme proposed, and GM-CBMeMBer filtering algorithm is used in the target recognition process.

The significant characteristics of wireless sensor networks determine that congestion often occurs [11-12]. Because the data transmission distance between sensor node and sink node is far, it is judged that the main way of data transmission in wireless sensor network is multi hop and multi to one communication mode. Wireless channel realizes resource sharing with the help of sensor nodes. When packets receive sensing events, they will quickly aggregate in a short time to further seize the limited broadband resources. Fierce conflicts will occur in the packets and pile up to the buffer area one after another, which will increase the possibility of network congestion [13]. Especially, the closer the distance from the base station is, the more likely congestion will occur. The decisive factor of global channel quality is the packet loss of congestion nodes in the buffer, which also leads to the delay problem, resulting in the lack of scientific application of resources [14-15]. Therefore, fair control algorithm can be used to effectively solve the congestion problem of wireless sensor networks.

To measure the computational complexity and target position estimation accuracy, node cooperative program is proposed. In 2007, Mahler proposed objective function posteriori expected number of targets (PENT) for the multiple target nodes management based on CPHD filter [16]. Maximized PENT can maximize the number detected by target, and can estimate the accuracy of the target state to a lesser extent [17-18]. In the system of this paper, CBMeMBer filtering algorithm is applied, and PENT function is applied in $\mathrm{CH}$ election. At each time step, as nodes of several clusters are activated, this paper also puts forward the corresponding data fusion method, and synchronous updating method and sequential updating method are respectively applied for WCSN of each level.

\section{SYSTEM FUNDAMENTAL}

\section{A. Node Rate}

When the nodes that meet the requirements can not be obtained, it can effectively reduce the data transmission probability of upstream nodes according to the scientific proportion, and ensure the close relationship between all nodes and sink nodes on the basis of each node sending the same packet weight. For example, nodes 1, 2, 3 and 4 are all source nodes. The data packets are transmitted to nodes 1, 2 and 3 one by one, and three data packets are transmitted to node 4 at the same time. When the congestion problem occurs in node 7 , it is necessary to slow down the speed of information transmission in time, so as to effectively solve the congestion 
problem [19]. Only in this way can each node have a fair chance to transmit data to sink. We can use the idea of fairness control for reference, and effectively combine the weight ratio of data flow in the process of channel allocation to solve the above problems.

At the same time, different data flows will pass through the same node, and several data flows may pass through a link $i, j$ at the same time. Through the link $i, j$, the sum of the rates of all the data streams can be expressed by $R_{i, j}$, and the overall rate of all the data streams through $i$ point is expressed by $R_{j}$.

$d(s)$ represents the data flow rate formed by a node.

$$
\begin{gathered}
r_{i, j}=\sum_{s \in N} r_{i, j}(s) \\
r_{i}=\sum_{s \in N} r_{i}(s)
\end{gathered}
$$

$W(i)$ represents the weight of data flow formed by node $i$. Let $F_{i}$ pass through the sum of all data stream weights of node $i$, and the calculation formula is as follows.

$$
F_{\mathrm{i}}=\sum_{s \in N} \mathrm{f}_{i}(s)=\sum_{s \in N} \frac{r_{i}(s) \times w(s)}{d(s)}=\sum_{k \in U_{i}} F_{k, i}(s)+w(i)(3)
$$

Where, $d(\mathrm{~s})$ is the propagation rate, that is, the delay and packet loss in the process of node forwarding. Formula (3) represents the weight of all data flows through node $i$, that is, the sum of node data flow weight generated by point $i$ and its own data weight. The upstream and downstream nodes of $i$ can be represented by $U_{i}$ and $D_{i}$. The weights of upstream and downstream nodes generated at $i$ point can be expressed as $k \in$ $U_{i}, j \in D_{i}, F_{k j}$ and $F_{i j}$.

$$
\begin{gathered}
F_{k j}=\sum_{s \in N} f_{k j}(s)=\sum_{s \in N, s \neq i} f_{k j}(s)=\sum_{s \in N, N \neq i} \frac{r_{k j}(s) \times w(s)}{d(s)}(4) \\
F_{i j}=\sum_{s \in N} f(s)=\sum_{s \in N} \frac{R_{I J}(s) \times W(s)}{d(s)}=F_{i} \frac{r_{i j}}{r_{i}}
\end{gathered}
$$

Set the initial values of $F_{i}, F_{k j}$ and $F_{i j}$ to 0 . In the process of calculating $F_{i}$, Formula (1) is applied specifically. In calculating the value of $F_{i j}$, Formula (5) and $i$ can be used to send $F_{i j}$ to node $j$, so as to better carry out the next calculation. Similarly, $i$ can receive $F_{k j}$ upstream node $k$. After repeated calculation, a numerical value is formed between $F_{i}$ and $F_{k j}$, and the stability is high. Once the $i$ node is congested, the channel is allocated to $k, i$ according to Formula (6).

$$
l_{k i}=\frac{F_{k i}}{F_{i}} r_{i}
$$

If point $i$ is the source node, then Formula (7) is used to allocate the channel reasonably for the local node.

$$
l_{i}=\frac{w(i)}{F_{i}} r_{i}
$$

\section{B. Target Signal}

For the convenience of description, the definition of network model is proposed. The upstream nodes can be represented by $U_{i}$. Information entropy means that each node $x$ sends a packet with an average amount of information. The calculation formula is as follows.

$$
H(x)=-\sum_{\mathrm{i}=1}^{\mathrm{q}} \mathrm{P}\left(\mathrm{a}_{1}\right) \log _{r} P\left(a_{i}\right)
$$

Among them, the amount of information refers to the size of all data packets obtained by detecting sensor nodes to remove redundant information. The amount of information in a data packet can be expressed by $a_{i}$, and the value of $q$ possibilities generated by $a_{i}$ can be expressed by $q$, and the basic probability corresponding to $a_{i}$ value is $p\left(a_{i}\right) . r$ is the unit of information entropy $H(x)$.

If the sensing data is redundant in WSN, the joint information entropy can be used to represent the joint information between nodes. Joint information entropy refers to the association between the amount of information in multiple data sources,

$$
H\left(X_{1} X_{2} \ldots X_{N}\right)=-\sum_{i_{N}=1}^{q} \ldots \sum_{i_{N}=1}^{q} P\left(a_{i 1} a_{i 2} \ldots a_{i N}\right) \log P\left(a_{i 1} a_{i 2} \ldots a_{i N}\right)(9)
$$

In real environment, some of the assumptions may not be always true, and the interference may affect measuring accuracy. There are two forms of expression about target signal $E_{i}(t)$, and the expression is shown in Formula(10) and Formula (11).

$$
E_{i}(t)=\frac{1}{N} \sum_{n=0}^{N-1} r_{i}^{2}\left(t+\frac{n}{f_{s}}\right)
$$

$$
E_{i}(t)=\frac{\gamma_{i}}{\left\|L_{T}(t)-L_{i}\right\|^{2}} E_{s}(t)+\varepsilon_{i}(t)
$$

Where, $E_{s}(t)=\frac{1}{N} \sum_{n=0}^{N-1} s^{2}\left(t+\frac{n}{f_{s}}\right), \varepsilon_{i}(t)=\frac{1}{N} \sum_{n=0}^{N-1} v_{i}^{2}\left(t+\frac{n}{f_{s}}\right)$.

$E_{s}(t)$ is subordinate to the Gauss random distribution $E_{s}(t) \sim N\left(E S, \sigma_{s}^{2}\right)$, and $\sigma_{s}^{2}$ is variance. As $v_{i}^{2}\left(t+n / f_{s}\right)$ is two order Chi-Square random variables.

\section{Multi-TARget Detection}

\section{A. Nodes in Sleep Mode}

$T_{d c}$ is defined as the sum of the wake-up time $\left(T_{o n}\right)$, and the sleep time( $\left.T_{\text {off }}\right) . D C$ is the ratio of $T_{o n}$ and $T_{d c}$. The $T_{o n}$ and $T_{\text {off }}$ can be obtained by Equation (12) and Equation (13),

$$
\begin{gathered}
T_{d c}=T_{o n}+T_{o f f} \\
D C=\frac{T_{o n}}{T_{d c}}
\end{gathered}
$$

\section{B. Persistent Object Detection Performance}

If a node is at point $A$, its area for perception can be expressed $\pi R_{s}{ }^{2}$. The probability $P$ can be expressed by Equation (14). 


$$
P=\frac{\pi R_{s}^{2}}{A}
$$

As $T_{\text {exist }}$ of persistent object is longer than $T_{d c}$, the object can be firmly detected if it is in the $R_{s}$ of a node.

According to Equation (14), Equation (15) can be obtained.

$$
D P=1-e^{-\lambda P}=1-e^{-d \times A \times \frac{\pi R s^{2}}{A}}=1-e^{-d \pi R s^{2}}
$$

It can be seen that $D P$ is determined by perceived distance and As the perceived distance increases, $D P$ can expand. $D P$ change with different $d$ can be obtained by setting $T_{d c}=5 \mathrm{~s}$. When $R_{s}$ is 4 meters and $d$ is $0.02, D P$ is about $50 \%$. But when $R_{s}$ is 4 meters and $d$ is $0.12, D P$ is nearly $100 \%$, as shown in Fig. 1.

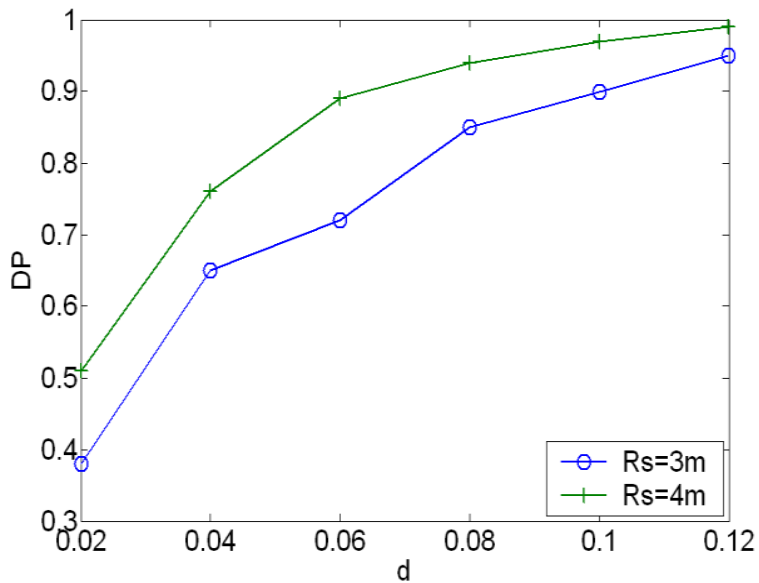

Fig. 1 Relationship of detection probability and $d$

Setting $T_{d c}=5 \mathrm{~s}$ and observe $D P$ change with different $R s$. The simulation results are similar to Fig.1. $D P$ increases with $R_{s}$. When $R_{s}$ increases, nodes can monitor a larger area, as shown in Fig. 2.

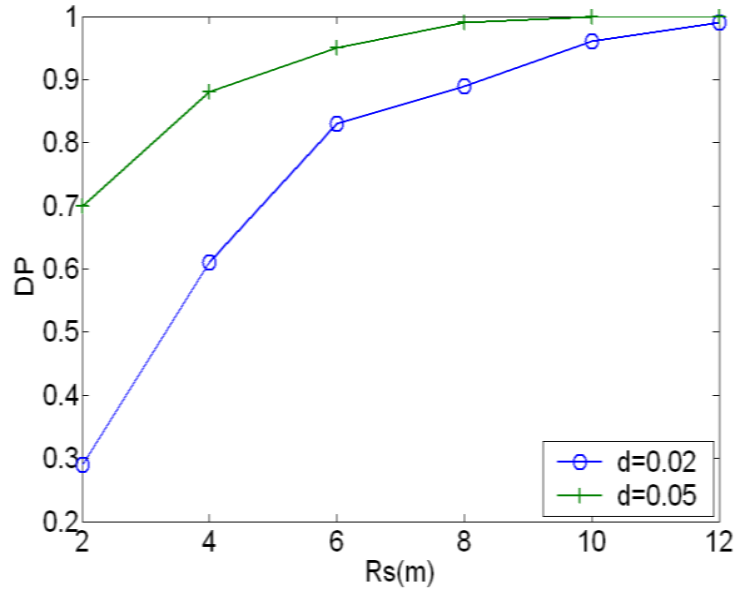

Fig. 2 Relationship of detection probability and $R_{s}$

As is known to all, the time delay $T D$ is much less than $T_{d c}$. If an object happens, the average detection delay can be denoted as Equation (16).

$$
T D(k)=\frac{T_{d c}}{2 \times(k+1)}
$$

When setting $R_{s}=5 \mathrm{~m}, T D$ decreases with $d$. A lower $d$ means less nodes to monitor the surveillance area, as shown in Fig. 3.

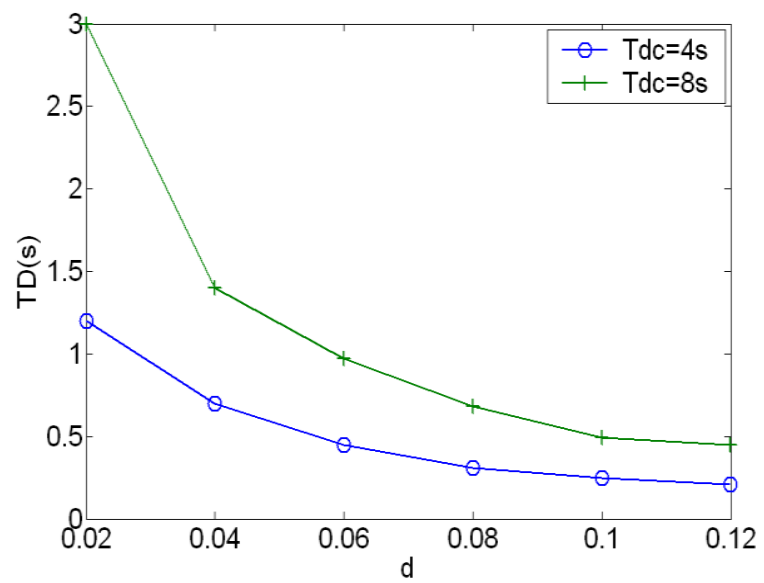

Fig. 3 Relationship of detection delay and $d$

As shown in Fig. 4, if $R_{s}=5 \mathrm{~m}, T D$ will change with $T_{d c}$, and this change is reflected in growth.

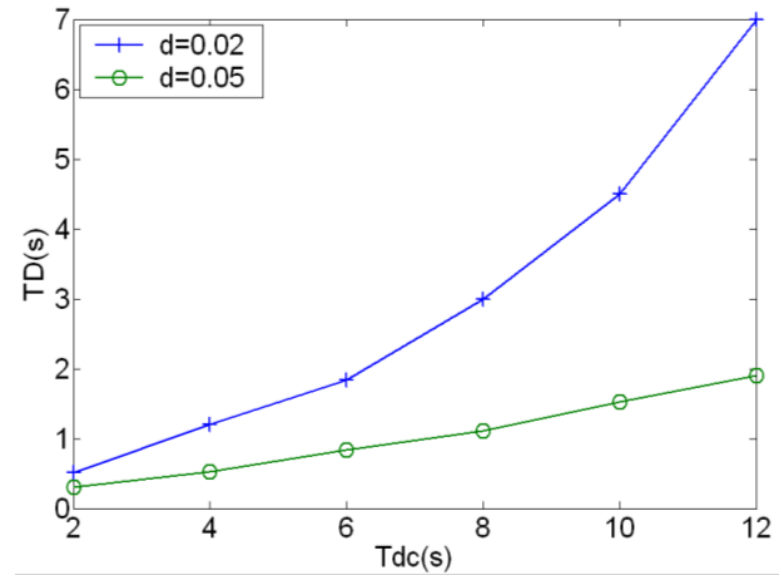

Fig. 4. Relationship of detection delay and $T_{d c}$

\section{Ephemeral Object Detection Performance}

The maintenance $T_{\text {exist }}$ should intersect the active time of node, and the temporal relationship is shown from Fig. 5.

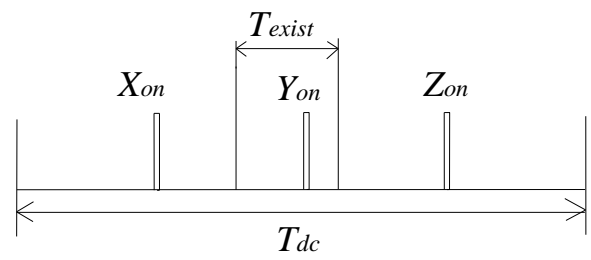

Fig. 5 The temporal relationship

The probability that $T_{\text {exist }} \leqslant T_{d c} / k$ is 


$$
\sum_{k=0}^{T_{d c} / T_{\text {exist }}} \frac{e^{-\lambda} \times \lambda^{k}}{k !}
$$

In this situation, the detection probability of ephemeral object can be denote as,

$$
\operatorname{DPE}\left(k_{1}\right)=\sum_{k=0}^{T_{d c} / T_{\text {exis }}} \frac{e^{-\lambda} \times \lambda^{k}}{k !}\left(1-(1-P)^{k}\right) \frac{k T_{\text {exist }}}{T_{d c}}
$$

On the contrary, when $T_{\text {exist }}>T_{d c} / k$, the detection probability is,

$$
\operatorname{DPE}\left(k_{2}\right)=\sum_{k=T_{d c} / T_{\text {exist }}}^{\infty} \frac{e^{-\lambda} \times \lambda^{k}}{k !}\left(1-(1-P)^{k}\right)
$$

So, the total detection probability is,

$$
D P E=D P E\left(k_{1}\right)+D P E\left(k_{2}\right)
$$

\section{SIMULATION}

This paper applies a two-level WCSN and randomly deployed nodes to deal with tracking pad and energy tracking in a cluttered environment. Initial target state is set as $X_{0}=[250,250,0,0]^{T}$ and $X_{1}=[-250,-250,0,0]^{T}$.

When probability $p_{S, k}=0.98$, existing target can survive. For simplicity, in this case, there is no value increase in this example. New spontaneous value increase of objectives depends on the Poisson point process when the intensity function is

$$
\gamma_{k}=0.1 N\left(\cdot ; m_{\gamma}{ }^{(1)}, P_{\gamma}\right)+0.1 N\left(\cdot ; m_{\gamma}{ }^{(2)}, P_{\gamma}\right)
$$

in which,

$$
\begin{aligned}
& m_{\gamma}{ }^{(1)}=[150,150,0,0]^{T}, \quad m_{\gamma}^{(2)}=[-150,-150,0,0]^{T} \quad, \\
& P_{\gamma}=\operatorname{diag}(100,100,25,25) .
\end{aligned}
$$

The sensor model is applied to simulate, and the parameter value is assigned as follows.

$$
F=\left\|\left[x_{k}, y_{k}\right]^{T}-L\right\|
$$

$S_{0}=291.34 n J, b=61.45 n J$, measurement noise

$\varepsilon \sim N\left(\square, 0, R_{k}\right) \quad, \quad R_{k}=\operatorname{diag}\left(\sigma_{\theta}^{2}, \sigma_{\delta}^{2}\right) \quad, \quad \sigma_{\theta}=0.0001$, $\sigma_{\delta}=2 n J, r=50, \theta=\pi / 4$.

The detection probability of nodes located within the sensing range of video node can be modeled as follows.

$$
p_{D, k}=p_{D, \max }-0.03 \times F / 400
$$

In the formula, $p_{D, \text { max }}$ is the best detection probability that can be achieved. Clutter is uniformly distributed in the monitoring area $-[-\pi / 2, \pi / 2] \mathrm{rad} \times[61.45,291.34] \mathrm{nJ}$, each camera is distributed with $\mathrm{r}$ points, that is, clutter RFS density is $k=r /(291.34 \pi)$. Then, depending on different clutter strength and the best detection probability, the simulation results can be drawn.

In addition, the performance of the system proposed for evaluation needs to obtain a measure of average performance. As it is obtained from [20], the total variation distance can be the miss distance of multiple targets. The total variation distance of any non-empty $\operatorname{subset}(\stackrel{\Lambda}{X}, X)$ is defined as

$$
d_{p}(\stackrel{\Lambda}{X}, X)=\min _{C} \sqrt[p]{\sum_{i=1}^{|X|} \sum_{j=1}^{|X|} C_{i, j}\left\|\hat{x}_{i}-x_{j}\right\|^{p}}
$$

Among them, the minimum value is received by transfer matrix $C$. The application of average Wasserstein miss distance is used as performance criteria.

Fig. 6 shows the relationship of network life and $T_{d c}$.

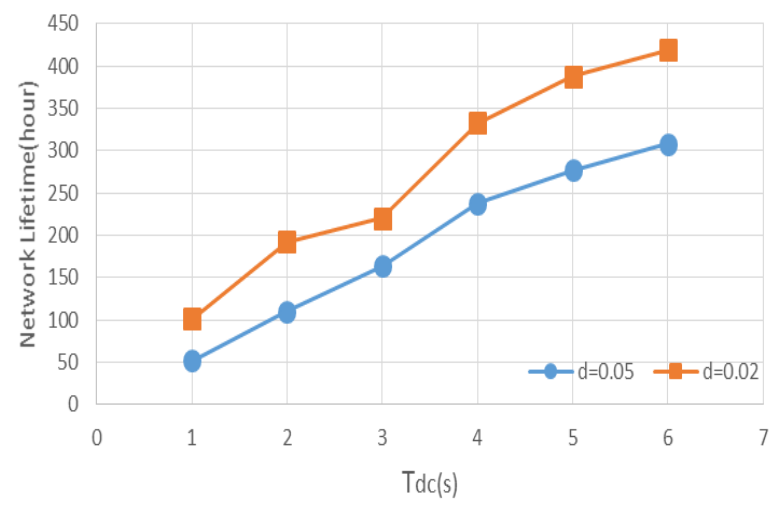

Fig. 6 Network life and $T_{d c}$

Fig. 7 shows the relationship of $d$ and $T_{d c}$.

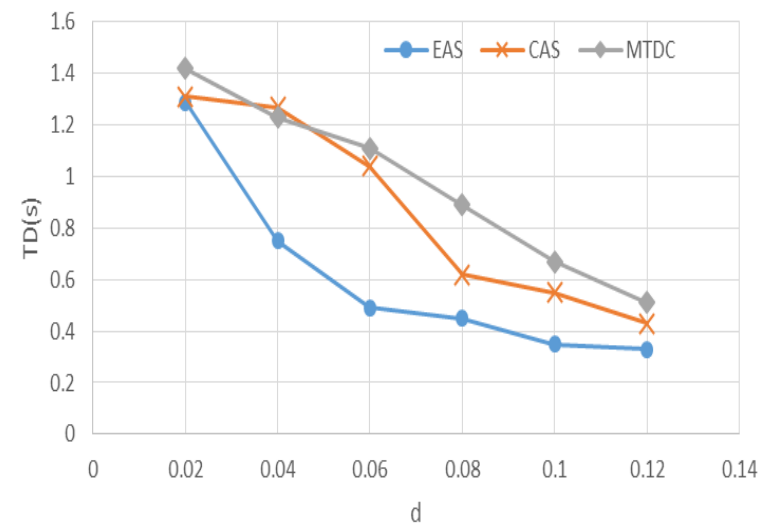

Fig. 7 Relationship of $d$ and $T_{d c}$

Figure 8 shows the relationship of work time and standard deviation of residual energy.

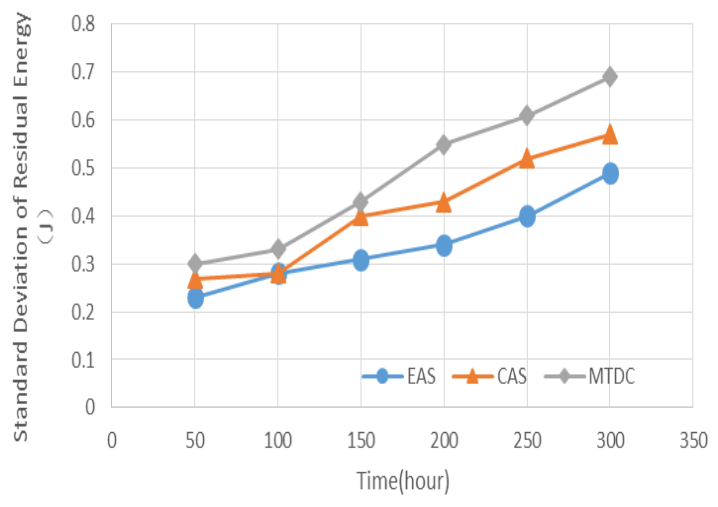


Fig.8 Relationship of work time and standard deviation of residual energy

Figure 9 shows network life of EAS, CAS and MTDC.

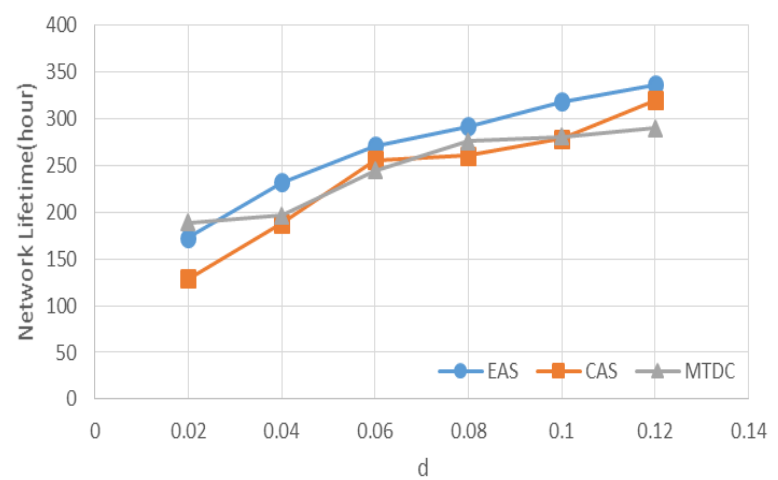

Fig. 9 Network life of EAS, CAS and MTDC

It can be obviously seen from the simulation results that this node cooperative program using particle CBMeMBer filtering algorithm can perfectly handle multi-target tracking, even if the sensor model is seriously nonlinear. Simulation results show that the tracking - forecasting data association scheme applying GM-CBMeMBer, which is proposed in this paper, runs well in identifying multiple target state, and can improve the estimation accuracy of multiple target state.

\section{CONCLUSION}

This paper presents a two-level WCSN to deal with MMTT problem, in which the number of tracked targets is unknown and time-variant.This paper proposes the election scheme of cluster head and cluster members and the data fusion method using particle CBMeMBer filtering algorithm.This paper proposes a tracking - forecasting association scheme to determine multiple target state estimation and associated target index. By using PCBMeMBer filtering algorithm, multiple target state RFS is obtained at each time step.In order to obtain the trajectory of a single target, this paper uses tracking forecasting association scheme to integrate target information.

In addition, the scheme proposed in this paper is more efficient in computational complexity and estimation accuracy, and this scheme is obtained through GMCBMeMBer filtering algorithm. The simulation results show that when the target's movement and the measurement process are severely nonlinear, the proposed architecture can still work. Therefore, the association method proposed in this paper is computationally efficient, and very appropriate for the condition that the prediction accuracy of target state is not very high.

\section{REFERENCES}

[1] Amgoth, T.; Jana, P.K. Energy-aware routing algorithm for wirless sensor networks. Computers \& Electrical Engineering, 2015,v 41:35767.

[2] Buranapanichkit, Dujdow; Deligiannis, Nikos; Andreopoulos, Yiannis convergence of desynchronization primitives in wireless sensor networks. IEEE Transactions on Signal Processing, 2015,v 63, n 1:221233.
[3] Changlin Yang; Kwan-Wu Chin. On complete target coverage in wireless sensor networks with random recharging rates IEEE Wireless Communications Letters, 2015,v 4, n 1:50-53.

[4] Ghosal, A.; Halder, S. Intrusion. Detection in a tailor-made gaussian distribution wireless sensor networks. Distributed Computing and Internet Technology. Proceedings: LNCS 8956:325-30.

[5] Iqbal; Anindya; Murshed, Manzur. A hybrid wireless sensor network framework for range-free event localization. Ad Hoc Networks, 2015,v 27:81-98.

[6] Leu, Jenq-Shiou, Chiang, Tung-Hung, Yu, Min-Chieh, Su, Kuan-Wu. B. Liu and D. Towsley. Energy efficient clustering scheme for prolonging the lifetime of wireless sensor network with isolated nodes. IEEE Communications Letters, 2015, v 19, n 2:259-262.

[7] T. Santhi Vandanna, S. Venkateshwarlu, K. Viswanath, Robust and Highly Secure Technique for Wireless Body Sensor Network using Sequence of ECG Data, WSEAS Transactions on Information Science and Applications, Volume 17, 2020, Art. \#17, pp. 138-145.

[8] Misra, Sudip; Mali, Goutam; Mondal, Ayan Distributed topology management for wireless multimedia sensor networks: exploiting connectivity and cooperation. International Journal of Communication Systems, 2015,v 28, n 7:1367-1386.

[9] Vaishali, Sandeep Santosh, User Association with RAP for Heterogeneous Wireless Railway Networks, WSEAS Transactions on Communications, Volume 18, 2019, Art. \#22, pp. 162-170.

[10] C. Gui and P. Mohapatra. Power Conservation and Quality of Surveillance in Target Tracking Sensor Networks. Proc. Of MobiCom, 2004: 129-143.

[11] P. Brass. Bounds on Coverage and Target Detection Capabilities for Models of Networks of Mobile Sensors. ACM Trans. On Sens. Netw., v $3, \mathrm{n} 2,9-17$

[12] R. Tan, G. Xing, B. Liu, J. Wang, and X. Jia. Exploiting Data Fusion to Improve the Coverage of Wireless Sensor Networks. IEEE/ACM Trans. On Netw., 2012, v20, n2: 450-462.

[13] Ayako Arao, Hiroaki Higaki, Clock Synchronization Algorithm Between Wireless Sensor Nodes without Additional Control Message Exchanges, WSEAS Transactions on Communications, Volume 18, 2019, Art. \#2, pp. 8-16.

[14] H. Zhang, C. Jiang, R. Hu and Y. Qian. Self-Organization in Disaster Resilient Heterogeneous Small Cell Networks. IEEE Network, 2016, v30, n2: 116-121.

[15] Q. Wu, M. Zhu, N. Rao, Integration of sensing and computing in an intelligent decision support system for homeland security defense, Pervasive and Mobile Computing, 2009, v5, n2: 182-200.

[16] H. Zhang, C. Jiang, X. Mao , Hsiao -Hwa Chen. Interference -Limit Resource Optimization in Cognitive Femtocells with Fairness and Imperfect Spectrum Sensing. IEEE Transactions on Vehicular Technology, 2016,v65, n3: 1761-1771.

[17] Asma Enayet, Md. Abdur Razzaque, Moving Target Tracking through Distributed Clustering in Directional Sensor Networks, Sensors, 2014, v14, n12, 24381-24407.

[18] Khushboo Jain, Anoop Bhola, An Optimal Cluster-Head Selection Algorithm for Wireless Sensor Networks, WSEAS Transactions on Communications, Volume 19, 2020, Art. \#1, pp. 1-8.

[19] P. Misra, S. Kanhere, D. Ostry, S. Jha. Safety assurance and rescue communication systems in high-stress environments: A mining case study. IEEE Communications Magazine, 2010, 5:1-8.

[20] Santoso, Fendy. Range-only distributed navigation protocol for uniform coverage in wireless sensor networks. IET Wireless Sensor Systems, 2015, v 5, n 1:20-30.

[21] Wang, Jiliang. On the delay performance in large scale wireless sensor network: measurement, analysis, and implications. IEEE/ACM Transactions on Networking, 2015, v 23, n 1:186-197.

[22] Wendi B. heinzelman, Anantha P. chandrakasan. An applicationspecific protocol architecture for wireless mirosensor networks. IEEE Transaction on wireless communication, 2002, v 1, n 4:660-670

\section{Creative Commons Attribution License 4.0 (Attribution 4.0 International, CC BY 4.0)}

This article is published under the terms of the Creative Commons Attribution License 4.0

https://creativecommons.org/licenses/by/4.0/deed.en_US 